Meta

Journal des traducteurs

Translators' Journal

\title{
Lexicographie bilingue (français-espagnol) et traduction : l'exemple
}

\section{Xavier Blanco}

Volume 42, numéro 1, mars 1997

Lexicologie et terminologie

URI : https://id.erudit.org/iderudit/002092ar

DOI : https://doi.org/10.7202/002092ar

Aller au sommaire du numéro

Éditeur(s)

Les Presses de l'Université de Montréal

ISSN

0026-0452 (imprimé)

1492-1421 (numérique)

Découvrir la revue

Citer cet article

Blanco, X. (1997). Lexicographie bilingue (français-espagnol) et traduction : l'exemple. Meta, 42(1), 133-141. https://doi.org/10.7202/002092ar

\section{Résumé de l'article}

On étudie les traductions d'exemples dans les dictionnaires bilingues et on examine, à partir d'un corpus quantitatif et d'une typologie des divergences de traduction, le type d'informations traductionnelles fournies par le lexicographe par le biais des exemples, et les difficultés rencontrées par l'utilisateur qui décode cette information. 


\title{
LEXICOGRAPHIE BILINGUE (FRANÇAIS-ESPAGNOL) ET TRADUCTION: L'EXEMPLE'
}

XAVIER BLANCO

Université Autonome de Barcelone, Barcelone, Espagne

\begin{abstract}
Résumé
On étudie les traductions d'exemples dans les dictionnaires bilingues et on examine, à partir d'un corpus quantitatif et d'une typologie des divergences de traduction, le type d'informations traductionnelles fournies par le lexicographe par le biais des exemples, et les difficultés rencontrées par l'utilisateur qui décode cette information.
\end{abstract}

\begin{abstract}
This article looks at the translation of examples in bilingual French-Spanish dictionaries. Based on a quantified corpus and a typology of translation differences, it discusses: the kind of translation information given by the lexicographer and the difficulties that the user may encounter in decoding this information.
\end{abstract}

\section{INTRODUCTION}

Quand bien même on admettrait (dans une perspective minimaliste) que la fonction principale d'un dictionnaire bilingue consiste (Zgusta 1971: 294) à mettre en rapport des unités lexicales de L1 (ou langue de départ) avec des unités lexicales de L2 (ou langue cible), équivalentes du point de vue de la sémantique lexicale, il n'en resterait pas moins vrai que, le plus souvent, l'usager a recours au dictionnaire bilingue non pour s'intéresser à l'équivalence «d'un mot dans une autre langue», mais pour mener à bien des tâches de décodage (compréhension), d'encodage (production) ou de traduction de formes appartenant à un discours. Cela sans compter que la dissymétrie essentielle des langues fait que la simple mise en relation d'unités de L1 avec des unités de L2 ne présenterait qu'un intérêt limité si elle n'était pas appuyée par toute une série de données complémentaires qui permettent de tirer profit des correspondances mises en évidence.

Ces données - c'est là l'objet de notre réflexion - sont présentées au moyen de différentes catégories d'information lexicographique qui sous-tendent la description du lemme et de ses équivalents de traduction. Dans cet article, nous nous intéresserons à deux de ces catégories : l'exemple (que nous appellerons aussi «séquence L1») et la traduction de l'exemple (ou «séquence L2»). Dans ce cadre, nous étudierons les divergences de traduction existantes entre les séquences $L 1$ et L2 en nous concentrant sur les applications possibles de cette recherche dans la tâche de rédaction du dictionnaire bilingue et, tout particulièrement, dans la sélection des exemples eux-mêmes.

Nous travaillerons à partir d'un corpus de base formé de 2580 exemples et de leurs traductions, tirés de cinq dictionnaires bilingues français-espagnol. Le cas échéant, nous mettrons en rapport ces données avec 1490 exemples et leurs traductions, tirés de cinq dictionnaires bílingues espagnol-français, afin d'estimer dans quelle mesure la traduction de l'exemple du français vers l'espagnol diffère de la traduction de l'espagnol vers le français. 
Les exemples mentionnés constituent la totalité des séquences présentées sous le traitement de la lettre «f $f$ dans les ouvrages signalés ci-dessous :

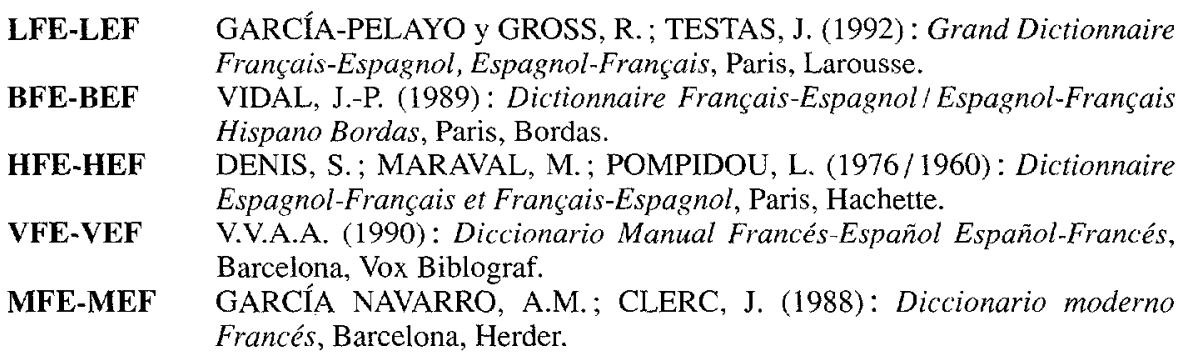

Par souci de brièveté, nous nous servirons désormais des abréviations indiquées en gras pour nous référer aux différents ouvrages. Au besoin, elles nous permettront de distinguer entre les dictionnaires français-espagnol (FE) et les dictionnaires espagnolfrançais $(\mathrm{EF})$.

Pour la représentation des structures des exemples, nous nous servirons des notations couramment utilisées au LADL (Paris 7) et au LLI (Paris 13), en l'occurrence:

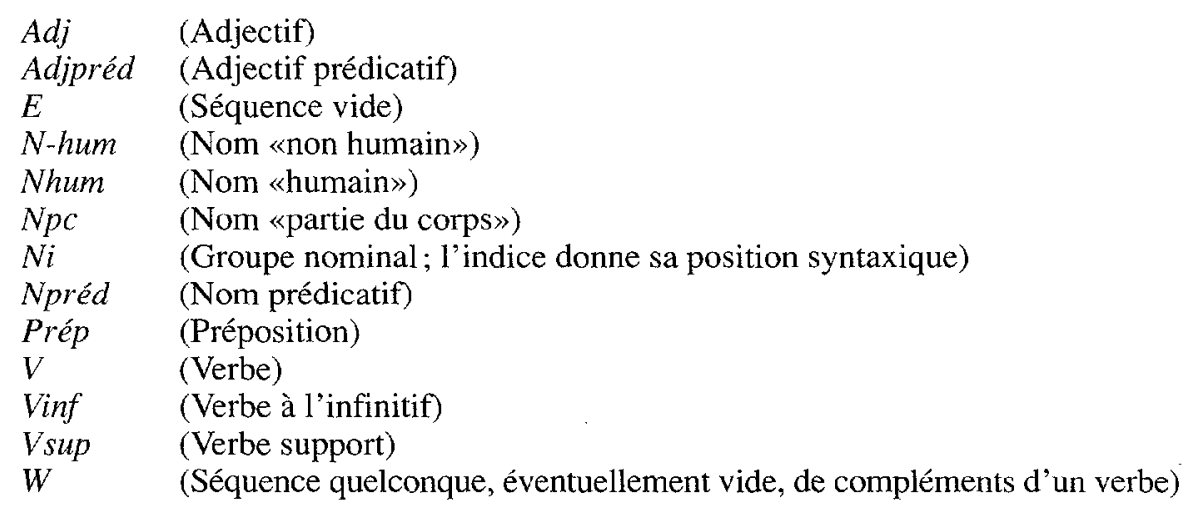

\section{TRADUCTION ISOMORPHE YS TRADUCTION HÉTÉROMORPHE}

Une première approche naive aux corpora d'exemples des différents dictionnaires permet de constater que certaines séquences reçoivent des traductions très proches de la langue source (p. ex. LFE avoir fâcheusement tendance à, tener desgraciadamente tendencia $a^{2}$; BFE faculté de droit, facultad de derecho), tandis que d'autres présentent des versions en L2 plus ou moins éloignées de la langue de départ (p. ex. LFE flûte à bec, flauta dulce; BFE il est en pleine possession de ses facultés, está en la plenitud de sus facultades), tout cela indépendamment de la traduction de la forme qui appartient au paradigme du mot vedette.

$\AA$ partir de cette simple observation, nous pouvons réaliser une première division dans le corpus. D'une part, nous qualifierons de «traductions isomorphes» les séquences L 2 dont les composants présentent une relation bijective avec ceux de la séquence L1, le sens lexical desdits composants étant semblable dans les deux langues ${ }^{3}$.

Pour ce qui est de ce genre de séquences, nous nous en tiendrons aux deux considérations suivantes:

$51,25 \%$ des séquences $\mathrm{L} 2$ considérées dans les dictionnaires français-espagnol sont des traductions isomorphes; 
- du point de vue de la traduction, l'apport informatif de ces séquences est limité. Cependant, la consommation d'espace qu'elles supposent dans le cadre de l'article de dictionnaire est importante (avec une longueur moyenne de 22,29 caractères supérieure à celle de la séquence $\mathrm{L} 1$, qui se situe à 20,16 caractères - la traduction de l'exemple constitue une catégorie d'information lexicographique très onéreuse). Il n'est donc pas surprenant que plusieurs métalexicographes aient suggéré leur élimination sous certaines conditions (Svensén 1993: 95).

D'autre part, nous appellerons «traduction hétéromorphe» une séquence L2 dont les composants ne présentent pas une relation bijective avec ceux de la séquence L1 ou si, même en remplissant la condition antérieure, elle contient un composant dont le sens lexical est nettement différent de celui de son homologue en L1. Dans les deux cas, nous dirons qu'il existe une divergence de traduction.

Nous pourrions considérer l'ensemble des traductions hétéromorphes comme le complémentaire de celui formé par les traductions isomorphes. Cependant, les traductions hétéromorphes ne constituent pas (contrairement aux traductions isomorphes) un ensemble homogène, car il est possible de distinguer plusieurs types de divergence de traduction par rapport à $\mathrm{L} 1$.

\section{DIVERGENCES DE TRADUCTION}

À l'instar de Françoise Vandooren (Vandooren 1993: 80), nous distinguerons sept cas de divergence de traduction (si l'on tient compte du dédoublement de la divergence de densité lexicale), que nous illustrerons moyennant des séquences L2 présentes dans les différents dictionnaires bilingues retenus:

- divergence syntagmatique : il existe une divergence syntagmatique quand un complément donné est actualisé par des syntagmes de différents types en L1 et en L2, normalement à cause de la sélection de l'élément prédicatif ou opérateur (p. ex. LFE fuir le danger, huir del peligro, où $\mathrm{L} 1=: \operatorname{Vinf} N_{1}$ et $\mathrm{L} 2=$ : Vinf Prép $\left.N_{1}\right)$;

- divergence catégorielle: il existe une divergence catégorielle quand le sens d'une unité lexicale donnée de L1 est lexicalisé en L2 par une unité lexicale appartenant à une catégorie morphosyntaxique différente (p. ex. LFE fouler le sol de la patrie, hollar el suelo patrio, où L1 =: Vinf $N_{1}$ Prép $N^{4}$ et $\mathrm{L} 2=: \operatorname{Vinf} N_{1} A d j$ );

- divergence lexicale: il existe une divergence lexicale quand une unité lexicale déterminée en L1 est rendue en L2 moyennant une unité qui ne présente pas un sens équivalent à la première si on les considère isolément (p. ex. HFE fièvre pourprée, fiebre maligna, où pourprée n'est pas mis en rapport avec purpúrea, mais avec maligna, une forme qui, considérée hors contexte, correspondrait à l'Adj français maligne);

a divergence de densité lexicale : il existe une divergence de densité lexicale quand un sens de L1 est rendu en L2 par un nombre différent d'unités lexicales. Nous aurons ainsi :

- divergence de densité lexicale par expansion (p. ex. LFE l'argent file, el dinero se gasta con rapidez, où $\mathrm{L} 1=: N_{0} V$ et $\left.\mathrm{L} 2=: N_{0} V A d v\right)$;

- divergence de densité lexicale par réduction (p. ex. VFE il faisait frire le poisson, él fréia el pescado, où $\mathrm{L} 1=: N_{0} V V_{i n f} N_{0}{ }^{l}$ et $\left.\mathrm{L} 2=: N_{0} V N_{l}\right)$;

- divergence thématique: il existe une divergence thématique quand les éléments prédicatifs de L1 et de L2 sélectionnent le même nombre et les mêmes classes d'arguments mais dans des positions syntaxiques différentes (p. ex. BFE mettre un prisonnier aux fers, poner grilletes a un preso, où Vinf $N$ hum, Prép $N$-hum s'oppose à Vinf N-hum 1 Prép Nhum 2 ); 
divergence prédicative: il existe une divergence prédicative quand la réalisation du prédicat en L1 et en L2 est inverse entre le verbe principal et un complément (MFE j'ai failli tomber, por poco me caigo, où faillir $-V$ - est rendu par por poco $A d v-)$.

Toutes les divergences signalées se trouvent dans notre corpus, mais leur importance quantitative est très variable. Il serait à remarquer, à cet égard, que la proportion de divergences de traduction lexicale et de divergences de densité lexicale par réduction s'accrôt sensiblement dans les dictionnaires qui affichent une tendance nette à se servir de la catégorie d'information lexicographique de l'exemple pour rendre compte de phénomènes de figement lexical (HFE et MFE). Cela est dû, d'une part, au fait que les suites figées ont très souvent recours à des images différentes selon les langues (p. ex. MFE dormir à poings fermés, dormir a pierna suelta), ce qui entraîne de nombreuses divergences lexicales. D'autre part, nombre de composés de L1 se voient attribuer des traductions univerbales (p. ex. HFE facteur receveur, estafetero), ce qui multiplie les divergences de densité lexicale par réduction. En fait, tout semble indiquer que le fait qu'un composé de LI présente une traduction univerbale en L2 va contribuer à le faire recenser par le lexicographe, qui sera plus sensible au caractère figé d'une unité complexe de Ll s'il constate qu'elle correspond à une unité simple en L2.

Les proportions des différents types de divergences de traduction dans notre corpus apparaissent dans le tableau ci-après.

\begin{tabular}{|l|c|c|c|c|c|}
\hline divergence/dictionnaire & LFE & BFE & HFE & VFE & MFE \\
\hline syntagmatique & $1,94 \%$ & $3,00 \%$ & $1,11 \%$ & $4,19 \%$ & $1,11 \%$ \\
\hline catégorielle & $2,52 \%$ & $2,55 \%$ & $3,50 \%$ & $2,39 \%$ & $2,51 \%$ \\
\hline lexicale & $3,88 \%$ & $4,05 \%$ & $16,08 \%$ & $3,59 \%$ & $20,83 \%$ \\
\hline expansion & $4,66 \%$ & $4,95 \%$ & $5,25 \%$ & $2,39 \%$ & $4,09 \%$ \\
\hline réduction & $2,52 \%$ & $5,40 \%$ & $16,08 \%$ & $2,99 \%$ & $19,44 \%$ \\
\hline thématique & $0,58 \%$ & $1,50 \%$ & $0,95 \%$ & $1,19 \%$ & $0,37 \%$ \\
\hline prédicative & $0,19 \%$ & $0,90 \%$ & $0,79 \%$ & $0 \%$ & $0,37 \%$ \\
\hline ISOMORPHISME & $83,68 \%$ & $77,62 \%$ & $56,21 \%$ & $83,23 \%$ & $51,25 \%$ \\
\hline
\end{tabular}

Figure 1:

Proportions des divergences de traduction

Précisons que l'inventaire des divergences de traduction existantes dans les dictionnaires espagnol-français qui font pendant aux ouvrages signalés ci-dessus offre des proportions semblables (Blanco 1995: 1065). Les différences les plus marquées s'observent précisêment dans le cas où le dictionnaire français-espagnol et le dictionnaire espagnolfrançais présentent, en raison de contraintes éditoriales, une certaine assymétrie (HFEHEF). Cette dernière observation, liée aux différences importantes quant au nombre de traductions isomorphes entre les dictionnaires appartenant à différentes maisons d'édition 
(jusqu'à 32,43\% d'écart entre le LFE et le MFE), fait penser que les chiffres indiqués obéissent davantage à des facteurs d'organisation du travail lexicographique qu'au sens de la traduction (français-espagnol vs espagnol-français).

\section{4. À TITRE D'ILLUSTRATION}

Comme illustration des apports possibles de l'étude des divergences de traduction dans le dictionnaire bilingue, nous aborderons quelques cas concrets en précisant à chaque fois comment la considération des relations traductologiques L1-L2 peut s'avérer utile non seulement pour préciser le contenu des exemples et la forme de leurs traductions, mais aussi pour juger de la pertinence d'un exemple déterminé sous une entrée déterminée.

\subsection{SOUHAITER VS FELICITAR}

Nous nous centrerons, d'abord, sur les exemples suivants :

HFE souhaiter la fête à qqn, felicitar a alguien por su santo

VFE souhaiter la fête à quelqu'un, felicitar a alguien por su santo

L'ordre linéaire des séquences lui-même permet déjà d'apprécier la divergence thématique. Le verbe de L1 sélectionne un premier complément sans préposition (fête) dont l'équivalent en L2 (santo) correspond au deuxième complément du verbe L2, précédé par la préposition por. En revanche, l'équivalent de traduction du deuxième complément de la séquence L1 (à qqn) correspond au premier complément de L2 (alguien). Nous

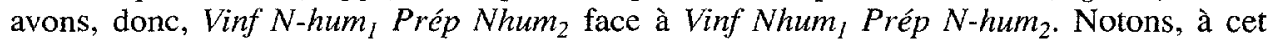
égard, que la préposition qui apparaît devant alguien n'est pas régie par le verbe felicitar. Elle obéit à une règle de caractère général selon laquelle les $N_{I}$ humains en espagnol sont précédés de la préposition $a$, ce qui doit permettre de les différencier du $N_{0}$, qui est souvent elliptique.

Cette sorte de chiasme entre les compléments régis par les verbes de L1 et de L2 donne parfois lieu à des irrégularités dans la rédaction même des exemples. Nous trouvons ainsi, dans le Larousse français-espagnol, la séquence suivante:

LFE souhaiter à quelqu'un sa fête, felicitar a uno por su santo

L'exemple, qui véhicule en fait la même information que ceux du HFE et du VFE, présente la particularité de renverser l'ordre canonique des compléments L1. En effet, $s a$ fête $\left.(N \text {-hum })_{l}\right)$ occupe la deuxième position après le verbe, tandis que à quelqu'un (Prép $\left.\mathrm{Nhum}_{2}\right)$ se voit déplacé vers la première position. Il s'agit d'un phénomène singulier, car l'ordre canonique des compléments est respecté dans les autres exemples du même ouvrage qui présentent la même structure :

\section{LFE faire sa montre à quelqu' un, limpiarle el reloj a uno \\ LFE flanquer la frousse à quelqu' un, meter miedo o darle mieditis a alguien \\ LFE frayer la voie à quelqu' un, abrir camino a alguien} $\ldots$

On pourrait en conclure que l'inversion observée obéit à l'existence de la divergence thématiques. Or, à notre avis, cette inversion non seulement n'est pas utile à l'usager, mais peut l'induire en erreur en masquant les difficultés qu'entraîne la traduction de souhaiter par felicitar.

Par ailleurs, et dans le même ordre d'idées, nous trouvons:

LFE prendre quelqu'un en filature, seguir los pasos de alguien

$\mathrm{HFE}$ prendre en filature (qqn), seguir la pista (de alguien)

MFE prendre en filature, seguir los pasos, la pista a alguien 
Il s'agit d'une situation similaire à la précédente, compte tenu que l'équivalent de traduction du Nhum $_{l}=$ : quelqu'un de prendre occupe la deuxième position après seguir (i.e. Nhum $_{2}=$ : alguien). Notons que, dans ce cas, le dictionnaire Larousse est le seul à ne pas cacher le problème, étant donné que le Hachette extrait le complément humain des deux séquences au moyen d'une parenthèse et que le Herder omet le complément humain de L1. Dans les deux cas, pour arriver à se servir convenablement de la traduction qui lui est proposée, l'usager devra d'abord prendre conscience de la divergence thématique, puis aller au-delà d'une présentation qui, paradoxalement, l'oblitère.

\subsection{FONDRE EN SUEUR VS SUDAR A MARES}

Les divergences prédicatives illustrent bien les différences de fonctionnement existant entre des prédicats L1 et L2 qui présentent pourtant un sens lexical équivalent. Examinons le cas suivant:

\section{MFE fondre en sueur, sudar a mares}

La séquence L1 présente un Npréd (sueur), combiné avec une extension aspectuelle (fondre) de son Vsup (être en). Étant donné que le $N$ espagnol sudor n'a pas de Vsup usité (la scule possibilité, exhalar, n'apparaît que dans des usages techniques), la traduction par sudar oblige à rendre l'aspect intensif au moyen d'un Adv (a mares).

La traduction proposée semble mettre en relief une certaine inadéquation de l'exemple lui-même sous l'entrée fondre. En effet, l'élément prédicatif de la séquence citée est sueur, fondre n'apportant au prédicat que, d'une part, les caractéristiques morphologiques verbales et, d'autre part, l'aspect intensif. S'agissant d'une collocation, elle devrait donc être présentée s.v. sueur, tout comme le Vsup non marqué être en.

D'autre part, l'analyse des séquences à actualisateur être Prép (être en, mais aussi être de ou être à) reste controversée. Face à la solution communément adoptée, à savoir, Vsup Npréd, où Vsup =: être en et Npréd =: sueur), il serait possible de décrire ces structures comme Vsup Adj, où Vsup =: être et $A d j=:$ en sueur. Plusieurs raisons semblent pouvoir être invoquées, qui justifieraient une telle analyse (Gross, sous presse). En effet, sueur, à la différence de la plupart des Npréd entrant dans des constructions Vsup Npréd:

- se pronominalise par le (p. ex. Luc est en sueur et Léa l'est aussi) et non par y ou en; - ne peut pas fonctionner comme tête de phrase relative (p. ex. *la sueur en laquelle est Luc);

a peut apparaître en position d'épithète ou d'apposition (p. ex. Le visage en sueur, Luc arriva; Luc, tout en sueur, arriva).

L'exemple du MFE introduit donc, s.v. fondre, des complexités d'autant plus embarrassantes qu'elles n'appartiennent pas à la description de ce verbe comme élément prédicatif.

\subsection{FRIRE VS FREIR}

Les divergences de densité lexicale par réduction offrent également quelques cas intéressants. Ainsi, nous avons:

BFE faire bouillir, faire chauffer, faire cuire, faire fondre, etc, hervir, calentar, cocer, derretir, etc.

Dans cet exemple du Bordas, le causatif disparaît dans la traduction, ce qui, en première analyse, n'a rien de surprenant si l'on tient compte du fait qu'il s'agit de verbes «culinaires», des verbes neutres par excellence aussi bien en espagnol qu'en français. Des séquences comme Luc fait bouillir le lait et Luc bout le lait sont donc en relation parasynonymique, tout comme Luc hace hervir la leche et Luc hierve la leche. 
Cependant, cette propriété doit être déterminée pour chaque unité lexicale aussi bien en L1 qu'en L2, car elle est susceptible de varier pour des verbes appartenant à des langues différentes, même s'il s'agit d'équivalents de traduction. En effet, si la séquence L1 comporte, à côté de faire bouillir, les suites faire fondre, faire cuire et faire chauffer, en L2 des combinaisons comme hacer derretir ou hacer cocer seraient plus douteuses comme parasynonymes de derretir et cocer respectivement, alors qu'une suite comme hacer calentar introduirait déjà forcément un nouvel actant par rapport à calentar. La traduction de l'exemple cité n'établit pas de distinction à cet égard, étant donné qu'elle ne présente pas les constructions factitives en espagnol.

La situation est encore différente dans les exemples suivants, extraits du Vox Biblograf:

VFE il faisait frire le poisson, él freía el pescado

VFE le poisson était en train de frire, el pescado se freía

En effet, d'une part, dans la séquence il faisait frire le poisson, il n'est plus possible d'éliminer le factitif 6 , car frire est un verbe défectif qui ne possède pas de forme pour la troisième personne du singulier du prétérit imparfait. D'autre part, en espagnol, la solution él freía el pescado est retenue non seulement parce que freír n'est pas défectif, mais parce que ?él hacía freir el pescado apparaît comme douteuse. En effet, si l'on considère cuire au lieu de frire, aussi bien il faisait cuire le rôti que il cuisait le rôti seraient acceptables en français, mais en espagnol le factitif poserait des problèmes d'acceptabilité.

Par ailleurs, le deuxième exemple cité ci-dessus présente une structure clé pour ce qui est de la relation traductologique entre les verbes culinaires français et espagnols. La périphrase durative (nécessaire, encore une fois à cause de la défectivité de frire) cache, en effet, un problème de traduction de type plus général; à savoir, l'impossibilité de traduire des séquences comme le poisson frit par *el pescado frie, et la nécessité d'avoir recours à el pescado se fríe ${ }^{7}$. Inversement, ?le poisson se frit serait très douteux en français. frire:

Nous pourrions résumer la situation de la façon suivante, aussi bien pour le verbe

$$
\begin{aligned}
& \text { Français } \\
& \text { Luc frit le poulet } \\
& \text { Luc fait frire le poulet } \\
& \text { le poulet frit } \\
& \text { ?le poulet se frit }
\end{aligned}
$$

$$
\begin{aligned}
& \text { Espagnol } \\
& \text { Luc fríe el pollo } \\
& * \text { Luc hace freír el pollo } \\
& \text { *el pollo frie } \\
& \text { el pollo se fríe }
\end{aligned}
$$

que pour d'autres verbes culinaires:

Français
Luc cuit le poulet
Luc fait cuire le poulet
le poulet cuit
?le poulet se cuit

Des raisons morphologiques, qui touchent seulement le verbe frire, font que les séquences Luc (frire) le poulet et le poulet (frire) sont remplacées par Luc (faire) frire le poulet et le poulet (être en train de) frire dès que le paradigme de flexion de ce verbe ne peut prendre en charge une forme déterminée. Cela ne doit pas, cependant, voiler le vrai problème de traduction (touchant non seulement frire, mais de nombreux verbes sémantiquement apparentés), qui réside dans l'impossibilité de traduire mot à mot des séquences françaises comme Luc fait cuire le poulet ou le poulet cuit. 


\section{CONCLUSION}

Après ce parcours rapide par des exemples et leurs traductions, nous souhaiterions mettre en relief quelques aspects qui, à notre avis, justifient la réflexion sur les divergences de traduction dans le dictionnaire bilingue.

Signalons, d'abord, que l'étude des divergences de traduction introduites moyennant les séquences L2 pourrait s'avérer utile, autant pour opérer les choix de traduction les plus appropriés aux buts pédagogiques et/ou utilitaires du dictionnaire que pour l'évaluation de la pertinence des séquences L1 comme exemples. De façon incidente, un tel travail réalisé pour un ensemble significatif du lexique nous semble susceptible de nourrir la réflexion traductologique autour d'une paire de langues donnée, ce qui pourrait également, par contrecoup, enrichir les descriptions bilingues proposées.

Il serait aussi à remarquer que la caractérisation des relations traductologiques existantes entre les séquences $\mathrm{L} 1$ et $\mathrm{L} 2$ pourrait contribuer efficacement à une séparation nette et rigoureuse entre exemples à proprement parler et suites figées, séparation qui, à l'heure actuelle, n'est pas tranchée. La traduction pourrait être employée comme un des critères opératifs pour établir cette différenciation. Ainsi, le lexicographe pourrait présenter en qualité d'exemples des séquences comme LFE foire agricole, feria del campo ou LFE pays francophones, países de habla francesa, car, bien qu'il ait penché respectivement pour une divergence catégorielle et une divergence de densité lexicale par expansion (que ce soit pour des raisons stylistiques ou de fréquence), les solutions littérales (feria agrícola et países francófonos) restent possibles. En revanche, des cas comme VFE fil de fer, alambre ou HFE poire fondante, pera de agua ne tolèrent pas une traduction mot à mot et seraient à traiter dans une catégorie d'information lexicographique réservée aux $N$ composés.

Finalement, il serait à souligner qu'une prise de conscience de la part du lexicographe des différents types de divergences introduites par la traduction des exemples pourrait se manifester en aval par des indications prévenant l'usager des éventuels dangers de la traduction d'une unité lexicale L1 donnée par une unité L2 sémantiquement équivalente mais présentant des différences de fonctionnement particulièrement difficiles à cerner. Des cas comme ceux qui ont étể présentés en 4.1. pourraient ainsi recevoir un tout autre traitement.

\section{Notes}

1. Une première version de ce travail a fait l'objet d'une communication dans le cadre du $I V$ Coloquio de la Asociación de Profesores de Filología Francesa de la Universidad Española, Las Palmas de Gran Canaria, 15-17 mars 1995.

2. Nous ne nous prononçons pas ici sur la pertinence des traductions proposées.

3. Faisons remarquer que cette dernière condition ne concerne pas, par définition, les composants qui ne présenteraient pas un sens lexical plein (p. ex. les prépositions régies par un élément prédicatif).

4. Faisons remarquer que le deuxième $N$ de la séquence étant un complément du nom de $N_{l}$, il ne peut comporter d'indice par rapport à fouler.

5. Bien que d'autres explications restent possibles. On pourrait se demander s'il n'y a pas eu un réflexe «antiargotique».

6. Même si cela aurait été possible d'un point de vue sémantique, étant donné que, pour ce genre de verbes décrivant une opération culinaire, la différence entre transitif et factitif pour ce qui est du contrôle extérieur exercé sur le processus est presque nulle (Boons et al. 1976: 88)

7. Construction que Z. S. Harris qualifiait de «moyenne» (Harris 1970).

8. On pourrait l'accepter dans des contextes comme le poulet se cuit depuis une heure. 


\section{RÉFÉRENCES}

BLANCO, X. (1995) : El ejemplo en el diccionario bilingüe, Thèse de doctorat, Université Autonome de Barcelone. BOONS, J.P.; GUILLET, A. et Ch. LECLÈRE (1976): La structure des phrases simples en français $I$. Constructions intransitives, Genève/Paris, Droz.

GROSS, G. (sous presse) : «Prédicats nominaux et compatibilité aspectuelle».

HARRIS, Z. S. (1970): Papers in Structural and Transformational Linguistics, Dordrecht, D. Reidel.

SVENSÉN, B. (1993): Practical Lexicography. Principles and Methods of Dictionary-Making. Oxford/New York, Oxford University Press.

VANDOOREN, F. (1993): «Divergences de traduction et architectures de transfert», La traductique, Montréal, Les Presses de l'Université de Montréal, AUPELF-UREF; pp. 77-90.

ZGUSTA, L. (1971): Manual of Lexicography, The Hague, Mouton. 